\title{
SIGNIFICADO VALORATIVO Y EMOCIONAL DE LA PERTENENCIA A LAS REGIONES DE O'HIGGINS Y ARICA PARINACOTA ${ }^{1}$
}

\section{Evaluative and emotional meaning of belonging to the regions of O'Higgins and Arica Parinacota.}

\author{
Paula Reveco-Quiroz * \\ Claudia Zúñiga**
}

\begin{abstract}
RESUMEN
El antecedente de esta investigación es el proceso descentralizador chileno, del cual surge la idea de describir las identidades regionales de Arica-Parinacota y O'Higgins a través de los significados valorativo y emocional que estudiantes universitarios otorgan a su pertenencia regional. El enfoque es cualitativo de tipo descriptivo-interpretativo, utilizando grupos focales. La muestra es no probabilística e intencional, formada por estudiantes universitarios de diferentes carreras y género, de cada región. Se realizó análisis de contenido de los datos a través de software Atlas ti. La tradición teórica que sustenta esta investigación es la identidad social de Henry Tajfel y la auto-categorización del yo de John Turner.
\end{abstract}

Palabras clave: Identidad regional, identidad social, regionalismo, descentralización.

\footnotetext{
${ }^{1}$ Esta investigación forma parte de la línea de investigación en Identidades Territoriales de la Facultad de Ciencias Sociales de la Universidad de Chile, financiada por los proyectos del Fondo Nacional de Desarrollo Científico y Tecnológico (FONDECYT) N 11090119 y VID SOC 06/15-2.

* Departamento de Psicología, Universidad de Chile. Santiago, Chile. Correo Electrónico: preveco@u.uchile.cl

** Departamento de Psicología, Universidad de Chile. Santiago, Chile. Correo Electrónico: cczuniga@u.uchile.cl Artículo recibido el 4 de junio de 2012. Aceptado el 23 de octubre de 2013.
} 


\begin{abstract}
The background of this investigation is the Chilean's decentralizing process from which the idea of describing Arica-Parinacota and O'Higgins's regional identity emerges by means of the evaluative meaning and the emotional meaning that university students give to their sense of regional belonging. It has a descriptive-interpretative qualitative approach and focus groups were carried out. The sample is intentional and non-probabilistic and consisted of university students from different careers and gender of each region.
\end{abstract}

Keywords: Regional identity, social identity, regionalism, descentralization.

\title{
INTRODUCCIÓN
}

La presente investigación se enmarca en el proceso descentralizador chileno, el cual, de acuerdo con los diagnósticos actuales, no ha conseguido alcanzar los hitos esperados para su avance. Es así que, el último informe territorial sobre Chile de la OCDE (2009) señala que el país sigue estando altamente centralizado, tanto en términos demográficos como económicos.

Dicha situación desencadena un incipiente consenso político para otorgar mayor autonomía al nivel subnacional de los gobiernos regionales (SUBDERE, 2009a) y así, promover procesos endógenos de desarrollo, ya que de acuerdo con la evidencia internacional, las estructuras de gobierno descentralizadas operan adecuadamente si son conducidas y lideradas por los propios actores regionales, en función de sus proyectos, prioridades y relación con su propia identidad, cultura y proyección (SUBDERE, 2009b).

El presente estudio se propone indagar en la identidad regional de estudiantes universitarios de las regiones chilenas Arica-Parinacota y O'Higgins, regiones que han sido seleccionadas en función de dos criterios de contraste: un criterio geográfico de mayor lejanía o cercanía a la capital nacional; y un criterio sociocultural, relacionado con la creación de la región. O’Higgins se encuentra junto a la capital nacional y fue creada en la división territorial implementada desde el nivel central en 1974, sin que necesariamente sus límites territoriales correspondieran a una historia sociocultural vinculada a una geografía (Raczynski y Serrano, 2001). Arica-Parinacota, en cambio, ubicada en el extremo norte del país, fue creada en el año 2007 (INE, 2007) producto de demandas de la propia ciudadanía. 
La población objetivo seleccionada han sido los estudiantes universitarios de ambas regiones, y esta decisión se basa en dos razones: primero, porque son un grupo que en su mayoría ha nacido después de la creación de las regiones, y a diferencia de sus padres no ha conocido otra forma de división territorial; y en segundo lugar, porque se ha considerado que este grupo sociodemográfico es uno de los que más posibilidades tiene de liderar futuros procesos de descentralización política y fortalecimiento de las identidades regionales.

Desde el punto de vista teórico, hemos elegido como marco de referencia la tradición de la identidad social, formada por la Teoría de la Identidad Social (TIS) y la Teoría de la Auto-Categorización del Yo (TAC), ya que, tal como señalan Scandroglio, López y San José (2008) han contribuido a la comprensión de la dimensión social de la conducta, en el marco de la Psicología Social contemporánea.

Desde esta tradición, se define la identidad social como: "Aquella parte del autoconcepto de un individuo que deriva del conocimiento de su pertenencia a un grupo (o grupos) social junto con el significado valorativo y emocional asociado a dicha pertenencia” (Tajfel, 1984: 292).

En este marco nos hemos planteado como pregunta de investigación si los habitantes de las regiones de Arica-Parinacota y O'Higgins tienen conciencia de pertenencia a sus regiones, y de ser así, cuáles son los significados valorativos y emocionales asociados a esta pertenencia..

\section{ANTECEDENTES TEÓRICOS}

\subsection{Identidad Social}

Tajfel (1981) estudia la auto imagen de los individuos que deriva de su pertenencia a grupos o categorías sociales, contribuyendo con el supuesto que ningún grupo vive aislado en la sociedad sino que todos los grupos viven en medio de otros. Ahora bien, cuando Tajfel habla de grupo, se refiere a un grupo psicológico, es decir, que ha de poseer tres características: a) una percepción por parte del individuo de que pertenece al grupo; b) la conclusión de que la pertenencia a ese grupo recibe una evaluación social positiva o negativa y de una cierta intensidad; y c) un cierto tipo de afecto asociado a la conciencia de la pertenencia grupal (Morales, 1999: 81). En consecuencia, la identidad social puede derivar de una gran variedad de pertenencias grupales, incluyendo las basadas en la etnia, el género, ocupación o la región en que se vive. Pertenecer a 
un grupo psicológico promueve el proceso de comparación social, el cual busca la diferenciación respecto de otros grupos y una distintividad positiva (Peris y Agut, 2007).

En este sentido, cuando la pertenencia a un grupo contribuye a alcanzar una distintividad positiva, el sujeto tenderá a permanecer como miembro del grupo, pero en el caso contrario, cuando la distintividad alcanzada por pertenecer a un grupo es negativa, el sujeto tenderá a desarrollar estrategias que le permitan mejorar su autoconcepto. En tal caso, Tajfel (1981) propuso dos tipos fundamentales de estrategias: La primera denominada movilidad social, que se basa en la creencia de que las barreras entre las categorías sociales son permeables, y consiste en que el sujeto redefine su pertenencia categorial, intentando pertenecer a un grupo de mayor estatus. La segunda, denominada cambio social, se relacionaría con la creencia en la impermeabilidad de las barreras intergrupales (la imposibilidad relativa de pasar, en términos psicológicos, de un grupo de estatus inferior a otro de estatus superior) y consistiría en una estrategia endogrupal conjunta para obtener una valoración positiva del mismo.

Dentro de la categoría de cambio social, Tajfel y Turner (1979, citado en Scandroglio, López y San José, 2008) propusieron originalmente dos tipos de estrategias fundamentales: creatividad social y competición social. La creatividad social tendría lugar cuando las relaciones intergrupales son subjetivamente percibidas como seguras; es decir: legítimas y estables, y en la formulación inicial de la teoría incluía tres estrategias concretas: búsqueda de nuevas dimensiones de comparación, redefinición de los valores adjudicados a determinadas dimensiones y cambio del exogrupo de comparación. La competición social aparecería cuando se percibe la comparación entre los grupos como insegura y consistiría en intentar aventajar al grupo de mayor estatus en la dimensión consensuadamente valorada por ambos (Tajfel, 1981 citado en Scandroglio, López y San José, 2008).

Otro de los elementos relevantes en la comprensión de los procesos de identificación social es la categorización del yo. El proceso de categorización social se refiere a una representación cognitiva de la percepción del entorno social en grupos (Turner, 1990), que permite estructurar el entorno, a partir de la sistematización y la simplificación del mismo (Deschamps y Devos, 1996), formando un sistema jerárquico de clasificación, donde cada categoría se incluye por completo en otra, en diferentes niveles de abstracción. 
Según Turner (1990), hay al menos tres niveles de abstracción de categorización delyo importantes para el autoconcepto social:a) nivel superordenado de categorizaciones del yo como ser humano, basadas en características comunes compartidas con otros miembros de la especie en comparación con otras formas de vida (identidad humana), b) nivel intermedio de categorizaciones endogrupoexogrupo, basadas en semejanzas y diferencias sociales entre seres humanos que le definen a uno como miembro de determinados grupos y no de otros (identidad social), y c) nivel subordinado de categorizaciones personales del yo, basadas en diferencias entre uno, como individuo único, y otros miembros del propio grupo, que le definen a uno como persona individual específica (identidad personal).

A diferencia de la categoría humana y de la categoría personal (que son unívocas), el nivel social es complejo y múltiple, y puede incluir muchas categorías jerarquizables sobre la base del grado de abstracción de los agrupamientos a que se refiere (Salazar, 1996).

\subsection{Identidad Regional}

Si bien existen diversas definiciones acerca de lo que se entiende por identidad regional, en esta ocasión se definirá a partir de la Teoría de la Identidad Social (Tajfel, 1984; Tajfel y Turner, 1989), como: "aquella parte del autoconcepto de un individuo que está basada en su pertenencia a un grupo regional, junto con el significado valorativo y emocional asociado a dicha pertenencia" (Zúniga y Asún, 2003: 75), y cuando se habla de grupo regional se hace referencia a un grupo humano que comparte un territorio con todas sus características geográficas, demográficas y culturales. En este sentido, la identidad regional no es un concepto estático, que es dado, y que hay que conservar, sino por el contrario, esta identidad se crea mediante procesos de adaptación en el espacio y en el tiempo (Zúñiga y Asún, 2003).

Por lo tanto, la identidad regional alude a un sentimiento de pertenencia y a un sistema cultural de referencia. No existe grupo sin cultura, y la cultura, como expresión de la identidad, se transmite, aprende y reproduce mediante los procesos de socialización (Arévalo, 1998, citado en Zúñiga y Asún, 2003). En definitiva, se propone que la identidad regional se basa en la conjunción entre el medio físico (base ecológica), la continuidad histórica (base temporal) y la continuidad social (base cultural) (Lisón, 1997, citado en Zúñiga y Asún, 2004). 


\section{MÉTODO}

El enfoque utilizado fue cualitativo, de tipo descriptivo-interpretativo. La técnica de recolección de datos fue el dispositivo de grupos focales y el análisis de los datos se llevó a cabo mediante la técnica de análisis de contenido cualitativo, con el software Atlas ti.

La muestra fue no probabilística e intencional $y$ en palabras de Dávila (1993), respondió a un criterio de comprensión y pertinencia y no de representatividad estadística. Los participantes fueron 37 estudiantes universitarios

que participaron en 4 grupos focales. Uno de varones y uno de mujeres en cada región, con la siguiente distribución: 9 participantes en el grupo femenino de Arica; 8 en el masculino de Arica; 10 en el femenino de O'Higgins y 10 en el masculino de O’Higgins. Los participantes estudiaban carreras de distintas áreas del conocimiento en diferentes universidades públicas y privadas.

\section{PROCEDIMIENTO}

Los grupos focales se realizaron en las capitales regionales de las regiones estudiadas, y fueron guiados por dos moderadoras a través de una pauta de entrevista semi-estructurada, que incluía preguntas abiertas que pretendían ser gatilladoras de una discusión, como por ejemplo, "si tuvieras que describirte ante una persona que no te conoce ¿qué dirías de ti?”. Los grupos focales fueron grabados y transcrito en forma textual.

En la Fase Analítica cuatro integrantes del equipo de investigación leyeron las transcripciones de dos de los grupos focales; segmentando los textos en unidades temáticas, proponiendo una malla de códigos. Luego, dichos códigos fueron revisados por otros dos investigadores del equipo, quienes triangularon la información. Cada uno leyó dos grupos de la muestra y realizó modificaciones en el sistema de categorías hasta llegar a un conjunto de 42 códigos, separados en categorías referidas a diferentes aspectos de las identidades sociales.

El análisis con Atlas ti consistió en: 1) insertar los documentos primarios (transcripción de los grupos focales) en la unidad hermenéutica, 2) realizar lecturas sucesivas de los textos, 3) Codificar cada párrafo, de acuerdo con la pauta de códigos generada, 4) Categorizar los códigos bajo familias, de acuerdo a un criterio de semejanza o criterio común, 5) Reorganizar las categorías en un network o red de relaciones, y 6) Construir interpretaciones de los datos. 


\section{RESULTADOS}

Las identidades sociales más salientes, que aparecen mencionadas espontáneamente en el discurso de los participantes, fueron la identidad nacional, la identidad universitaria y la identidad regional. De acuerdo con los objetivos de investigación reportados, en este artículo sólo se analizará la identidad regional.

Los resultados se presentarán agrupados en torno a las semejanzas y diferencias entre ambas regiones y posteriormente se describirán los resultados distintivos y particulares de cada región.

- Conciencia de pertenencia a las regiones de Arica-Parinacota y O'Higgins. A partir del discurso de los participantes de ambas regiones se puede observar claramente su consciencia de pertenencia a su región, la que aparece en forma espontanea cuando se les pregunta cómo se describirían ante una persona que no sabe nada de ellos mismos. Más notoriamente aún, aparece la pertenencia a la respectiva capital regional, tal vez por ser el lugar de residencia de la mayoría de los participantes.

- Significado emocional asociado a la pertenencia a las regiones de AricaParinacota y O'Higgins. Las emociones asociadas a la pertenencia regional son fundamentalmente positivas, destacando el orgullo de pertenecer al propio grupo regional. Sin embargo, aparecen algunas diferencias no menores entre las regiones estudiadas, ya que en O'Higgins, los participantes declaran un profundo sentimiento de frustración por las condiciones de deprivación percibidas, y en Arica-Parinacota en cambio, aunque también aparece la percepción de falta de recursos, ésta no afecta el tono positivo de la emoción asociada a la pertenencia a la región.

- Significado Valorativo de la pertenencia a las regiones de Arica-Parinacota y O’Higgins. A pesar de tratarse de regiones tan diferentes en términos geográficos y socioculturales, el análisis de contenido de los discursos de los participantes ha dejado al descubierto importantes semejanzas en el significado valorativo que sus habitantes le otorgan a la pertenencia a su región. A continuación revisaremos dichas semejanzas:

- Abandono. En ambas regiones los participantes destacan el abandono como una categoría relevante de su pertenencia regional. En Arica-Parinacota se experimentaría como aislamiento e iría acompañado por una sensación de orfandad respecto de Chile, básicamente producto de la lejanía geográfica que mantiene con 
la capital nacional, pero sumado a una percepción de falta de interés por parte del centro del país. En el caso de O’Higgins, este abandono se experimentaría como invisibilización, vale decir un sentirse "borrados" en el contexto nacional, a partir de la excesiva proximidad con la capital nacional.

- Calidad de vida. Los participantes de ambas regiones destacan la tranquilidad y la seguridad que se vive en la región. Esta es una categoría valorada positivamente, que según señalan los participantes sería distintiva de su región en comparación con otros lugares del país, especialmente la capital nacional, Santiago, que es percibida con características opuestas.

- Limites sociales permeables. Esto se asocia al constante movimiento emigratorio que los participantes describen. En ambas regiones se identifica como un problema la pérdida de capital humano que les significa la migración de muchos coterráneos, fundamentalmente los más jóvenes, en busca de mejores oportunidades. Esta estrategia de abandonar el grupo, en busca de otro que confiera al individuo un mejor estatus, corresponde al mecanismo psicológico que Tajfel (1984) denomina movilidad social, los participantes reportan además, que un número importante de quienes emigran, finalmente regresan a la región, pudiendo suponerse que no resulta psicológicamente fácil abandonar el grupo regional, pues al parecer, a pesar del bajo estatus que otorga en el contexto nacional, esta pertenencia también proporciona satisfacciones, las que de acuerdo con los participantes, estarían relacionadas con su historia familiar, sus vínculos sociales, costumbres y tradiciones.

- Devaluación de la imagen regional. Los participantes de Arica-Parinacota reportan que la imagen que se muestra de la región en el resto del país, a través de los medios de comunicación, destaca los aspectos más negativos en lugar de los positivos, fomentando la imagen de que Arica es una región en crisis, que no progresa, o con problemas de delincuencia y narcotráfico. En el caso de O'Higgins, los participantes afirman que el problema de la imagen devaluada de la región tiene dos orígenes. Por una parte, que Rancagua es una ciudad de paso que no tiene atractivos turísticos que incentiven a las personas de otras regiones a quedarse en ella, y por otra, que existiría en el país una mirada despectiva hacia la figura del huaso, personaje típico de la región, que ellos valoran positivamente como un símbolo de su cultura, pero que desde fuera sería visto como una persona que no sabe hablar o vestir correctamente o que es excesivamente tímida. 
- Patriotismo. Tanto en Arica-Parinacota como en O'Higgins, los participantes perciben como un componente relevante de su propia identidad regional el patriotismo, el cual valoran positivamente, existiendo símbolos o hitos regionales que lo promueven. En el caso de Arica-Parinacota, el símbolo de este patriotismo sería fundamentalmente el Morro de Arica, así como el himno de la región y la conmemoración de la toma de Arica. En el caso de O’Higgins, se releva la conmemoración del hito histórico de la Batalla de Rancagua. Para los participantes de Arica-Parinacota resulta muy relevante destacar que a diferencia de muchos de los eventos históricos que se conmemoran en nuestro país, en la región se celebra una victoria, y hacen énfasis en esta distinción entre ganar o perder fundamentalmente porque de esa manera se distinguen positivamente de Iquique, ciudad que perciben como su rival, ya que el combate naval de Iquique, la efeméride más recordada en esta ciudad, lo que conmemora es precisamente una derrota. En O’Higgins, en cambio, los participantes señalan que lo relevante es que en su región ocurrió un hecho histórico de trascendencia para el país.

Contrastes en los significados valorativos de la pertenencia a las regiones de Arica-Parinacota y O'Higgins. Además de semejanzas, también se han encontrado contrastes en los significados entre participantes de las dos regiones, que se describen a continuación:

- Percepción de las concepciones fronterizas y sureñas. Los participantes de Arica-Parinacota señalan que existe una identificación fronteriza de su región, producto de su condición de región limítrofe con otros países, lo cual evalúan positivamente. Los participantes de O’Higgins por su parte, indican que existe discordancia entre la imagen que ellos tienen de su propia ubicación geográfica y la imagen que les atribuyen externamente desde otras regiones, lo cual evalúan negativamente, ya que ellos creen que se ubican en el centro del país, mientras que las personas de Santiago o de más al norte los etiquetan como parte del sur de Chile. Se desprende de estas afirmaciones que la categoría "sur" tiene una connotación negativa para los participantes, en comparación con la categoría "centro", dentro de la cual prefieren estar.

- Redes de contacto de cada región. Ambas regiones establecen redes de contacto con diferentes territorios aledaños, lo cual evalúan positivamente. En Arica-Parinacota los participantes reportan un contacto continuo con personas de los países limítrofes como Perú y Bolivia y algunos ubicados más al norte en América del Sur, de los cuales reciben, en sus propias palabras, positivas influencias 
culturales. Los participantes también destacan, en comparación, el poco contacto que mantienen con la región chilena más cercana, Tarapacá.

Por su lado, los participantes de O’Higgins, declaran mantener redes de contacto principalmente con la Región Metropolitana, y dicen reconocer los beneficios de estar cerca de la capital, la cual les provee de muchos de los servicios que su propia región necesita y de los cuales carece.

- Homogeneidad Social versus Estratificación Social. Los participantes de Arica-Parinacota señalan que en su región existe homogeneidad socio-económica entre sus habitantes, es decir, que la gran mayoría de la población correspondería a los que ellos llaman "clase media" y que no habría grupos con mayor poder adquisitivo. Esto lo evalúan positivamente, porque a su parecer generaría una forma particular de relacionarse al interior del grupo regional, más horizontal e inclusiva. Mientras que en el caso de O'Higgins, los participantes destacan la alta segmentación socio-económica que existe entre la población regional, generando dificultades en las relaciones intergrupales, lo cual es evaluado negativamente por los participantes. Esta notoria brecha en los ingresos se debería a la diferenciación entre quienes se desempeñan en la gran minería del cobre y quienes viven de labores agrícolas.

\section{Elementos distintivos de la pertenencia a la región de Arica-Parinacota}

- Cultura milenaria. Los participantes de Arica-Parinacota destacan la tradición cultural de más de 10 mil años que los identifica, y cuya trayectoria evidentemente trasciende a la reciente creación administrativa de la región. Este uno de los componentes que más orgullo genera en los participantes, generando distintividad positiva al endogrupo.

- Multiculturalidad. Otro de los elementos que los participantes subrayan es que la cultura de Arica-Parinacota se ha caracterizado por la integración en su territorio de distintas etnias y culturas, tales como aymaras, afrodescendientes, peruanos, bolivianos y europeos, y que esto se expresa a través de sus tradiciones, costumbres y herencia histórica cultural de sus habitantes.

- Diversidad geográfica. Los participantes destacan la diversidad geográfica como una de las características más sobresalientes de Arica-Parinacota, que se potencia a partir de dos factores como son la posición fronteriza de la región y el tamańo reducido de la misma. Esto permite trasladarse fácilmente de un extremo 
a otro de la región e incluso fuera del país, accediendo en corto tiempo a diferentes paisajes, por ejemplo al mar, la montańa o los valles.

- Bohemia. Los participantes reportan que la noche es un componente importante de su jornada diaria, ya que el clima caluroso incentiva que muchas actividades comiencen tarde. Así, destacan la vida nocturna como una característica positiva de la región, que se manifiesta en la diversidad de lugares para salir de noche y disfrutar de un ambiente de distensión.

- Rivalidad con la región vecina. Los participantes describen una conflictiva relación con la región de Tarapacá, personificada en su capital Iquique. Estas dificultades son explicadas por ellos como derivadas de una antigua competencia por recursos y reconocimiento, que data desde la época en que Arica era una provincia de Tarapacá, y que no fue resuelta con la creación de la nueva región.

\section{Elementos distintivos de la pertenencia a la región de O'Higgins}

- Tradición huasa. Los participantes destacan la importancia de la figura del huaso, que por un lado, se relaciona con la tradición agrícola de la zona y por otro, los hace percibirse a sí mismos como el prototipo del chileno. De acuerdo con los participantes de esta región, la figura del huaso es la que mejor representaría la imagen consensuada del chileno y por lo tanto es un orgullo para ellos que esta figura sea también el prototipo del habitante de su región.

- Tradición agrícola. Los participantes de O'Higgins valoran positivamente esta actividad económica característica de la región, ya que significa el reconocimiento de su propio territorio, de su fertilidad, de su capacidad productiva, de su capacidad turística y de la capacidad para generar puestos de trabajo.

- Tradición campesina. Relacionada con la tradición huasa y la tradición agrícola, aparece esta categoría que los participantes vinculan a las personas "de campo" que habitan las zonas rurales de la región y se caracterizan por vivir en contextos pequeńos, donde la mayoría de la gente se conoce y desarrollan relaciones humanas de mucha cercanía. La mística de las personas de campo se relacionaría con su calidad humana y el cariño que expresan agasajando a sus visitas con un trato especial. Los participantes reportan que en general la población que habita en la ciudad tiene familiares en el campo, lo que representa un lazo afectivo que los vincula con esta zona, de la cual se sienten orgullosos.

- Tradición minera. Aun cuando la minería no es la actividad productiva más desarrollada en la zona, ésta representa para sus habitantes un importante 
punto de referencia, que se relaciona con el estatus que la minería del cobre confiere a la región y a sus trabajadores, por ser uno de los elementos más importantes de la producción económica nacional. No obstante, también se crean conflictos a partir de las diferencias de estatus generadas por medio de esta actividad, ya que el nivel de ingresos de los trabajadores del cobre sería notoriamente más alto que el de quienes se desempeñan en las actividades agrícolas.

\section{CONCLUSIONES}

La presente investigación surgió a partir del interés por explorar si una categoría social, creada e impuesta externamente hace casi cuatro décadas, como es la región de O’Higgins, y una categoría de más reciente creación político administrativa, pero supuestamente más representativa de los intereses de los propios ciudadanos, como es la región de Arica-Parinacota, han sido asumidas por las personas e incorporadas a su propio autoconcepto, es decir, si se ha construido una identidad social vinculada a estos territorios.

Los resultados de este trabajo permiten concluir que, efectivamente, las regiones de O'Higgins y Arica-Parinacota son percibidas por nuestros participantes como categorías diferenciadas. Así lo demuestra el hecho de que la categoría regional aparezca en forma espontanea en el discurso de los participantes, cuando se les pide que se definan a sí mismos. Esto estaría indicando que los participantes pueden ver a los habitantes de su región como un grupo diferenciado y no sólo como una muestra aleatoria del grupo nacional más inclusivo.

Un elemento a destacar es que a partir del análisis de contenido del discurso de los participantes, no se aprecian diferencias interregionales en la intensidad de la identidad regional. Es decir, a pesar de que la región de O’Higgins fue creada a partir de un proceso arbitrario y lejano a sus propios habitantes, y Arica-Parinacota ha vivido un proceso de construcción más inclusivo; o visto desde otra dirección, a pesar de que O'Higgins tiene ya casi 40 años de historia como región reconocida oficialmente por el Estado y Arica-Parinacota es de muy reciente creación, los participantes de ambas regiones evidencian similares procesos de identificación social con su territorio.

Es relevante señalar además, que en general hubo concordancia entre los participantes de cada región al describir los elementos en los que se basa su identidad regional. Esto nos recuerda que hay una construcción social del significado de las identidades, que conduce a las personas a mostrar consenso en al menos algunos 
aspectos de su definición. Como plantea Deaux (2000), la interrelación entre la experiencia individual y el contenido socialmente comunicado, es una cuestión clave para el análisis de la identidad social.

Respecto al contexto social entonces, debemos recordar que las regiones chilenas no son categorías construidas en base a diferencias culturales o sociales evidentes, sino más bien al contrario, estas regiones describen sociedades que no reconocen grandes diferencias culturales y cuyos principales contrastes son las características geográficas y las grandes distancias entre algunas de ellas. Así lo indican los datos de la última Encuesta Nacional de Juventud (Instituto Nacional de la Juventud, 2009), que muestra que prácticamente no existen diferencias entre los jóvenes de distintas regiones, en términos culturales, valóricos ni conductuales, y el Informe Desarrollo Humano en Chile 2002 (Programa de las Naciones Unidas para el Desarrollo), que originalmente incluía un capítulo sobre culturas regionales, y que debió ser descartado debido a que no se encontraron datos significativos y consistentes sobre diferencias culturales interregionales.

Además, debemos considerar que al interior de las regiones existe gran diversidad étnica, cultural y económica. En O’Higgins, tal como los propios participantes relatan, existen estilos de vida, vinculados a sistemas productivos, muy diferentes e incluso, conflictivos entre sí. En Arica-Parinacota contrastan las comunidades indígenas del altiplano con la vida en los valles o junto a la costa.

Si tal como plantea Turner (1990), la formación de una categoría depende de la comparación de estímulos, y dentro de cualquier marco de referencia, es más probable que un grupo de estímulos se categorice como una entidad en la medida en que las diferencias entre estos estímulos se perciban como menores que las existentes entre éstos y otros estímulos, no deja de sorprender el que en un contexto de grandes diferencias intraclase, y menores diferencias interclase, como son las regiones chilenas, las personas de todos modos logren categorizarlas como entidades diferenciadas e identificarse con ellas.

Sin embargo, son los postulados del propio Turner los que nos permiten explicar esta situación, ya que, como el mismo señala, la saliencia de una categorización del yo conduce a la acentuación perceptiva de las semejanzas intraclase y de las diferencias interclase (Turner, 1990).

Así, que desde los inicios de la regionalización chilena se incluyeran en los planes de estudios y textos escolares las características distintivas de las regiones, enfatizando las diferencias geográficas y el funcionamiento administrativo del 
Estado a través de los representantes regionales del Gobierno Central, puede haber logrado, efectivamente, un aumento de la saliencia de la categoría, favoreciendo de este modo la percepción de distintividad en O’Higgins. En el caso de AricaParinacota, el proceso de creciente creación, la cobertura dada por los medios locales y las expectativas generadas, sin duda han contribuido a generar una mayor saliencia de esta categoría.

En síntesis, podemos señalar que hemos logrado los objetivos planteados de determinar si los participantes tienen conciencia de pertenencia a sus regiones y cuáles son los significados valorativos y emocionales asociados a esta pertenencia. La conciencia de pertenencia aparece claramente en el discurso de los participantes y se asocia a emociones de orgullo y calidez, pero también a una cierta resignación por el abandono percibido, y a una valoración que a pesar de considerar ciertos elementos negativos, brinda un saldo final positivo.

Este último elemento es relevante de desatacar, ya que el discurso de los participantes denota que algunas características de la región derivan en un bajo estatus dentro del contexto nacional. Tal como plantea Tajfel (1984), al menos en nuestros tipos de sociedades, los individuos intentan mantener una imagen satisfactoria de sí mismos. Por lo tanto, cuando las características objetivas o percibidas del grupo, no favorecen o dificultan mantener esta imagen positiva, el sujeto tenderá a abandonarlo o a desarrollar estrategias psicológicas que compensen esta situación.

Así, en el análisis de estos casos idiosincráticos como es la identificación con las regiones de O’Higgins y Arica-Parinacota, podemos concluir que a pesar de que los significados valorativos asociados a esta pertenencia contienen algunas características negativas, los participantes utilizan la estrategia de creatividad social y reinterpretan estas características de modo que se transforman en un aporte a la distintividad positiva de su región. De este modo, los participantes de Arica y Parinacota reconocen por ejemplo, que se sienten aislados del resto del país, pero consideran que esa condición es la que les permite estar más cerca de otros países y enriquecerse con el intercambio cultural; En el caso de O`Higgins, los participantes plantean por ejemplo, que su ubicación junto a la capital nacional los invisibiliza frente al resto del país, pero a la vez señalan que esta misma ubicación les permite acceder a todo el desarrollo, la conectividad y los servicios que la capital ofrece.

Finalmente, amerita resaltar que los resultados de este estudio estarían evidenciando no sólo una positiva valoración de la pertenencia a su región por 
parte de los participantes, sino también de su pertenencia al estado Chileno. Esto puede desprenderse de su reclamo explícito ante lo que ellos perciben como abandono por parte del resto del país. Su demanda por una mayor atención hacia sus regiones no es sólo una aspiración de mayor entrega de recursos desde el Estado central, sino fundamentalmente, de un mayor grado de reconocimiento.

En el caso de O'Higgins este reconocimiento aparece asociado a identificar como imagen prototípica del chileno, la figura del huaso, que sería representativa a la vez de la región. En el caso de Arica-Parinacota debiera considerar el orgullo de haber defendido al país exitosamente durante la Guerra del Pacífico y continuar ejerciendo ese rol como ciudad frontera. La aspiración de los participantes de esta región es que Chile reconozca el rol fundamental que ejerce Arica en la protección de la soberanía nacional y que ese reconocimiento se exprese en los distintos ámbitos de la vida pública.

En cuanto a las limitaciones y proyecciones del presente estudio, el haber trabajado con población universitaria y con una muestra no representativa, no permite la generalización de los datos ni a otros sujetos de la misma población de estudiantes universitarios ni a otras poblaciones dentro de la región. Sin embargo, estos resultados pueden constituirse en hipótesis de trabajo para nuevas investigaciones sobre la materia, contribuyendo a la generación de conocimiento sobre la construcción de identidades sociales regionales en nuestro país

\section{REFERENCIAS}

Dávila, Andrés. "Las perspectivas metodológicas cualitativa y cuantitativa en las ciencias sociales: debate teórico e implicaciones fraseológicas". En J. M. Delgado y J. Gutiérrez (Eds.), Métodos y técnicas cualitativas de investigación en ciencias sociales. Madrid: Síntesis, (1993): 69-83.

Deaux, Kay. Models, meanings and motivations. En D. Capozza y R. Brown. Social Identity Process: Trends in theory and research. Londres: SAGE, (2000):1-14.

Deschamps, J. y Devos, T. Relaciones entre identidad social e identidad personal. En F. Morales, D. Páez, J. Deschamps y S. Worchel (Eds.), Identidad social: Aproximaciones psicosociales a los grupos y a las relaciones entre grupos. Valencia: Promolibro, (1996): 39-55. 
Instituto Nacional de Estadísticas. Boletín informativo. Enfoque estadístico: Nuevas regiones de Chile. Santiago de Chile, 2007. Disponible en: <http://www. ine.cl/canales/menu/boletines/enfoques/2007/pdf/INENuevasRegiones. pdf>. [Consultado: el 2 de mayo del 2010].

Instituto Nacional de la Juventud. VI Encuesta Nacional de Juventud. Santiago de Chile, 2009.

Morales, José Francisco. La identidad social. En A. Aguirre y J. F. Morales (Eds.), Identidad social y cultural. Barcelona: Bardenas, (1999): 79-112.

Organización para la Cooperación Económica y Desarrollo. Informe Territorial de Chile. Paris: Ministerio del Interior Chile, 2009.

Peris, Rosana y Agut, Sonia. "Evolución conceptual de la identidad social. El retorno de los procesos emocionales", Revista Electrónica de Motivación Emoción, X (26-27), (2007): 1-11. Disponible en: <http://reme.uji.es/ articulos/numero26/article2/article2.pdf>. [Consultado: el 20 de enero del 2011].

Programa de las Naciones Unidas para el Desarrollo. Informe Desarrollo Humano en Chile. Santiago de Chile, 2002.

Raczynski, Dagmar y Serrano, Claudia. (Eds.), Descentralización: Nudos Críticos. Santiago de Chile: Corporación de Investigaciones Económicas para Latinoamérica, 2001.

Scandroglio, Barbara, López, Jorge y San José, María Carmen. "La Teoría de la Identidad Social: una síntesis crítica de sus fundamentos, evidencias y controversias", Psicothema 20/1 (2008): 80-89.

Subsecretaría de Desarrollo Regional y Administrativo. Documento de sintesis y propuestas finales. Politicas para la descentralización: Construyendo institucionalidad para un Chile heterogéneo. Santiago de Chile: Ministerio del Interior, 2009a. Disponible en: http://www.subdere.gov.cl/1510/ articles-79573_archivo_fuente.pdf [Consultado: el 6 de enero del 2011].

Subsecretaría de Desarrollo Regional y Administrativo. Identidad Regional: 
Reconociendo la diversidad para el desarrollo de los territorios. Santiago de Chile: Ministerio del Interior, (2009b). Disponible en: <http://www. territoriochile.cl/1516/articles-79403_recurso_1.pdf>. [Consultado: el 16 de abril del 2010].

Tajfel, Henri. Human Groups and Social Categories. Cambridge: University Press, 1981.

- Grupos Humanos y Categorias Sociales. Barcelona: Herder, 1984.

Tajfel, Henri y Turner, John. La teoría de la identidad social de la conducta intergrupal. En J. F. Morales y C. Huici (Eds.), Lecturas de psicología social. Madrid: Universidad Nacional de Educación a Distancia, (1989): 41-87.

Turner, John. Redescubrir el grupo social. Madrid: Morata, 1990.

Salazar, José Miguel. Identidad social e identidad nacional. En J. F. Morales, D. Páez, y J. C. Deschamps. Identidad Social. Aproximaciones psicosociales a los grupos y a las relaciones entre grupos. Valencia: Promolibro, (1996): 495515.

Zúñiga, Claudia y Asún, Rodrigo. "Identidad regional en un contexto de cambio: un estudio en la Araucanía, Chile”, Psicología Politica 26 (2003): 73-92.

Zúñiga, Claudia y Asún, Rodrigo. "Diseño y Validación de una Escala de Identidad Regional”, Revista de Psicología Social 19 /1 (2004): 35-49. 